XIX IMEKO World Congress

Fundamental and Applied Metrology

September 6-11, 2009, Lisbon, Portugal

\title{
LIMITATIONS OF PRECISION LENGTH MEASUREMENTS BASED ON INTERFEROMETERS
}

\author{
Gerd Jaeger \\ Ilmenau University of Technology, Institute of Process Measurement and Sensor Technology, \\ P.O.Box 100 565, 98684 Ilmenau, Germany, gerd.jaeger@tu-ilmenau.de
}

\begin{abstract}
The most important principles, the basis and operation of heterodyne and homodyne interferometers are discussed. Their benefits and limitations are covered based on a metrological analysis. The resolution of interferometers can be determined by calculating the smallest resolvable distance increment. Also, it is shown here how the Abbe comparator principle can be fulfilled in all three measuring dimensions by using interferometers. Other factors in addition to the Abbe errors are discussed which affect the measurement uncertainty of interferometric length measurements.

The Abbe-error-free design is explained using the example of a nanopositioning and nanomeasuring machine developed at the Institute of Process Measurement and Sensor Technology (Ilmenau University of Technology) and manufactured at the SIOS Meßtechnik GmbH Ilmenau, Germany.
\end{abstract}

Keywords: Heterodyne and homodyne interferometers, metrological analysis, Abbe-error-free design, nanopositioning and nanomeasuring machine

\section{INTRODUCTION}

Today's nanometrology limits the accuracy of precision engineering. These limits are based on the metre definition as redefined in 1983 as well as on the comparison between an iodine-stabilised helium-neon laser and a stabilised helium-neon laser to be calibrated, on the influence of the refractive index of the air, on the realisation of the Abbe principle and so on. Laser interferometric devices are employed in precision measurements and positioning tasks, since they provide a means for attaining high metric resolution and precision, even over long measurement ranges. The versatility and broad applicability of laser interferometers are unattainable using any other metrological methods. Laser interferometers suitable for use in advanced areas of microtechnology, nanotechnology and precision engineering must have small measuring heads that are insensitive to environmental effects.

Single, double and triple beam plane mirror interferometers are applied in a nanomeasuring machine in order to measure and control the six degrees of freedom of the 3D-nanopositioning stage. Their metrological benefits are due to their compact dimensions and their utilisation of optical fibres for coupling the laser light source. It is shown now the Abbe principle can be realized in a nanomeasuring machine using plane mirror interferometers. Their benefits and limitations are covered based on metrological analysis.

\section{FUNDAMENTALS OF INTERFEROMETERS}

\subsection{The "Interference" in Interferometers}

The following section covers only interferometers with amplitude division. It is sensible to differentiate between interferometers with neutral beam splitters and ones with polarising beam splitters.

Homodyne interferometers usually use neutral beam splitters, whereas heterodyne devices require polarising elements.

The propagation of light is described by the Maxwellian wave equations. For homogenous isotropic non-conductors it holds $[1,2]$ :

$$
\begin{aligned}
\Delta \vec{E}-\frac{1}{v^{2}} \ddot{\vec{E}} & =0 \\
\Delta \vec{H}-\frac{1}{v^{2}} \ddot{\vec{H}} & =0
\end{aligned}
$$

where $\vec{E}$ is the electrical field strength vector, $\vec{H}$ the magnetic field strength vector, $v$ the phase velocity of the wave in a medium with the refractive index $n$ where $v=c_{0} / n$ and $\mathrm{c}_{0}$ the phase velocity of the wave in a vacuum.

If no birefringent medium is present, the propagation of the light can be described by equation (1). A solution to the equation is possible with the assumption of monochromatic light:

$$
\vec{E}(\vec{r}, t)=\vec{a}(\vec{r}) \cdot e^{j[\vec{k} \cdot \vec{r}-\varpi t+\vartheta]}
$$

$\vec{a}(\vec{r})$ is the vector amplitude, $\vec{k}=\frac{2 \pi}{\lambda} \cdot \vec{e}_{k}$ is the wave number vector, $\vec{r}$ the position vector and $\vartheta$ the phase for $\mathrm{t}=\mathrm{r}=0$.

Figure 1 shows the simplified set-up of a heterodyne interferometer. In order to calculate the intensity distribution $\mathrm{I}$, it is assumed that the polarising beam splitter (PBS) feeds beam $\omega_{1}$ into one interferometer arm and $\omega_{2}$ into the other arm. Furthermore, it is presumed that two planar, monochromatic, linearly polarised waves with frequencies $\omega_{1}$ and $\omega_{2}$ interfere. 


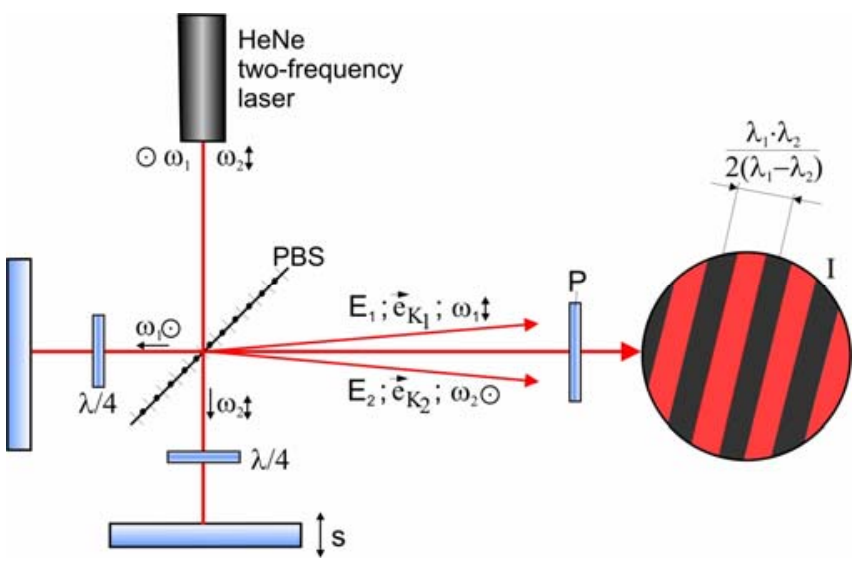

Fig. 1: Heterodyne Interferometer

The following relationships are used to calculate the intensity distribution:

$$
I=\frac{\varepsilon \cdot \varepsilon_{0} \cdot C}{2} \cdot E \cdot E^{*}=\frac{\varepsilon \cdot \varepsilon_{0} \cdot C}{2} a^{2}
$$

where $\mathrm{E}$ is a complex value, $\mathrm{E}^{*}$ its complex conjugate value, a the amplitude.

From

$$
E=\sum_{i} E_{i}
$$

with

$$
\begin{aligned}
& E_{1}(\vec{r}, t)=a_{1} \cdot e^{j\left[\vec{r} \vec{k}_{1}-\omega_{1} t+\vartheta_{1}\right]=j \gamma_{1}} \\
& E_{2}(\vec{r}, t)=a_{2} \cdot e^{j\left[\vec{r} \vec{r}_{2}-\omega_{2} t+\vartheta\right]=j \gamma_{2}}
\end{aligned}
$$

and

$2 \cos \left(\gamma_{1}-\gamma_{2}\right)=e^{j\left(\gamma_{1}-\gamma_{2}\right)}+e^{-j\left(\gamma_{1}-\gamma_{2}\right)}$

it follows that

$$
I_{p}=I_{1_{p}}+I_{2_{p}}+2 \sqrt{I_{1_{p}} I_{2_{p}}} \cos \left(\gamma_{1}-\gamma_{2}\right)
$$

(the subscript $\mathrm{p}$ denotes polarised)

$$
\gamma_{1}-\gamma_{2}=\gamma=\vec{r}\left(\vec{k}_{1}-\vec{k}_{2}\right)+\left(\omega_{2}-\omega_{1}\right) t+\vartheta_{1}-\vartheta_{2}
$$

The interference orders change with the frequency $\omega_{2}-\omega_{1}$, yielding a synthetic wavelength $\lambda_{\text {syn }}=\frac{\lambda_{1} \cdot \lambda_{2}}{\lambda_{1}-\lambda_{2}}$.

In contrast to heterodyne interferometers, homodyne interferometers operate using one frequency. Therefore, it holds for $\omega_{1}=\omega_{2}$ :

$$
\gamma=\vec{r}\left(\vec{k}_{1}-\vec{k}_{2}\right)+\vartheta_{1}-\vartheta_{2}
$$

The phase $\gamma$ only changes with a change in the optical path difference.

In the following section only homodyne interferometers will be discussed.
If an interferometer reflector is displaced a distance s, it follows that

$$
I=I_{1}+I_{2}+2 \sqrt{I_{1} I_{2}} \cos \left(\gamma+\gamma_{M}\right)
$$

and

$$
I=I_{1}+I_{2}+2 \sqrt{I_{1} I_{2}} \cos \left(\gamma+\frac{2 \pi}{\lambda_{0}} \cdot n \cdot i \cdot s\right)
$$

$\gamma$ describes the interference pattern before the reflector displacement and $\gamma_{\mathrm{M}}$ contains the phase change resulting from the movement of the reflector by s.

$\mathrm{i}$ is termed interferometer factor; the interferometer in fig. 1 possesses a factor $i=2$. From equation (10) it follows for the displacement $\mathrm{s}$ :

$$
s=\frac{k \cdot \lambda_{0}}{i \cdot n}=\frac{k \cdot c_{0}}{i \cdot n \cdot f_{H e N e_{s t a b}}}
$$

where $\lambda_{0}$ is the wavelength of light in vacuum, $\mathrm{k}$ the change of the interference order with a displacement $s$ of the reflector and $\mathrm{f}_{\mathrm{HeNestab}}$ the frequency of a stabilised $\mathrm{He}-\mathrm{Ne}$ laser.

\subsection{Metrological Analysis}

\subsubsection{Resolution}

Next, the smallest resolvable displacement $s_{\mathrm{q}}$ must be determined. If an interference order $(\mathrm{k}=1)$ is divided into $\mathrm{e}$ electronic increments, it follows from equation (11) that:

$$
s_{q}=\frac{\Delta k \cdot \lambda_{0}}{i \cdot n}=\frac{\lambda_{0}}{e \cdot i \cdot n}
$$

with $\Delta k=\frac{1}{e}$, where $\Delta \mathrm{k}$ is the smallest resolvable interference order and $\mathrm{e}$ is the number of electronic increments per interference order. Given that $\frac{\lambda_{0}}{n}=632.8 \mathrm{~nm}, \mathrm{i}=2$ and $\mathrm{e}=256, \mathrm{~s}_{\mathrm{q}}$ is then determined to be only $1.24 \mathrm{~nm}$.

\subsubsection{Uncertainty of measurement}

Some parameters affecting the uncertainty of measurement of $\mathrm{s}$ can be derived from equation (11). $\mathrm{c}_{0}$ :

The big advantage of the metre definition of 1983 is that the value of the speed of light in a vacuum was fixed at a value of $299792458 \mathrm{~m} / \mathrm{s}$.

$\mathrm{k}$ :

The number of impulses $\mathrm{N}$ counted during the displacement of a reflector by s can be calculated by equation 13 : 


$$
N=\frac{i \cdot e \cdot n}{\lambda_{0}} \cdot s
$$

Nonlinearities of the interpolation electronics must be considered when determining $\mathrm{N}$.

n:

With the help of the Edlen formula, the dependence of the refractive index of the air on air pressure, humidity and temperature can be compensated for within certain limits. The following relative uncertainty results from the empirically determined Edlen formular as well as from the measurement uncertainty of the sensors:

$$
\frac{\Delta n}{n}=5 \cdot 10^{-8}
$$

This means that measurements over long ranges $(>30 \mathrm{~mm})$ must take place in a vacuum, with a vacuum of $10^{-3}$ bar being sufficient.

$\mathrm{f}_{\text {HeNestab: }}$

The frequency $f_{\text {HeNestab }}$ must be known as indicated in equation (11). For this, the difference is determined between $\mathrm{f}_{\mathrm{HeNestab}}$ and the frequency of an iodine-stabilised He-Ne laser [3, 4], the frequency of which is coupled to the caesium frequency standard $\mathrm{f}_{\mathrm{cs} 133}$. The wavelength of the iodine-stabilised $\mathrm{He}-\mathrm{Ne}$ laser resulting from the traceable connection is $\lambda_{\mathrm{HeNe}}=632.99139822 \mathrm{~nm}$ with a relative standard uncertainty of $2.5 \cdot 10^{-11}$ [4] (see fig. 2).

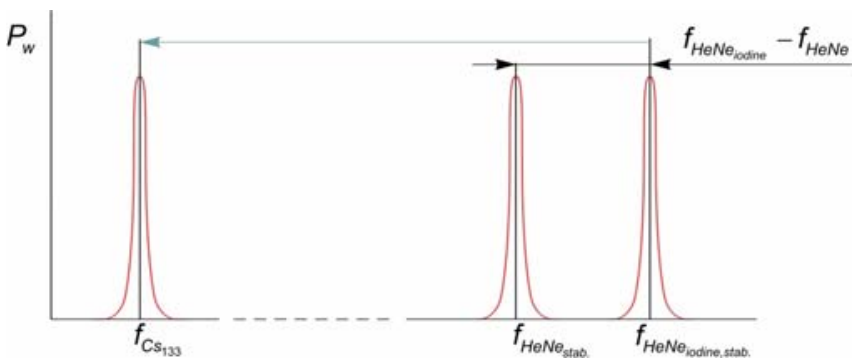

Fig. 2: Traceability of a stabilised He-Ne-Laser

Further influences affecting the measurement uncertainty of interferometric measurements must also be minded. Such influences can be caused for example by not observing the Abbe comparator principle or as a result of the instability of the metrological frame.

Both types of interferometers - i.e. those equipped with plane mirrors and those equipped with corner cube reflectors - are needed for applications in nanopositioning and nanomeasuring engineering. Only plane mirror interferometers allow the taking of three-dimensional coordinate measurements $[5,6]$.

\section{THE DESIGN OF PLANE MIRROR INTERFEROMETERS}

Figure 3 shows the difference between a plane mirror interferometer of the state of the art (above) and the interferometer developed by our institute (below) $[5,6]$. The most advantage of our plane mirror interferometer is that it has only one measuring beam. This fact is important for compliance with the Abbe comparator principle on all three measurement axes. The interferometer (above) needs two beams for having tilt invariance in a small angle range of the moving plane mirror.

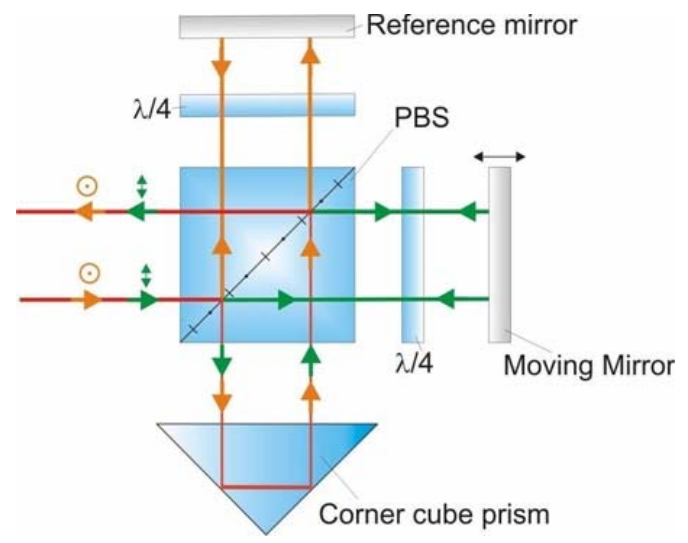

Plane mirror interferometer of the state of the art

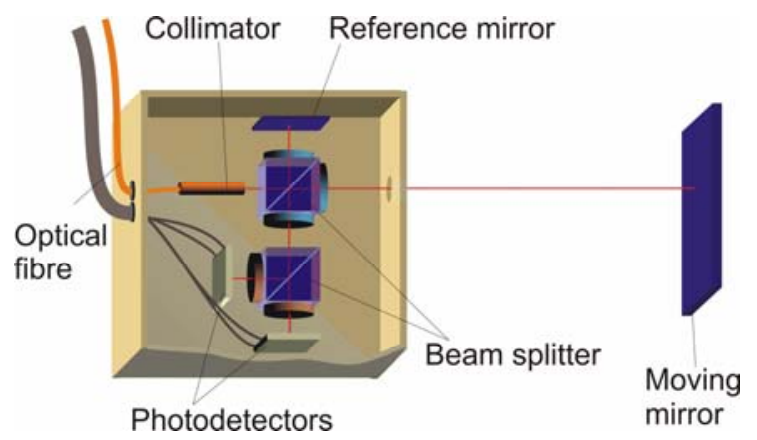

Plane mirror interferometer developed by the Institute of Process Measurement and Sensor Technology

Fig. 3: Plane mirror interferometers

Single, double and triple beam plane mirror interferometers can be applied in the nanomeasuring machine in order to measure and control the six degrees of freedom of the 3D-nanopositioning stage (see Fig. 4).

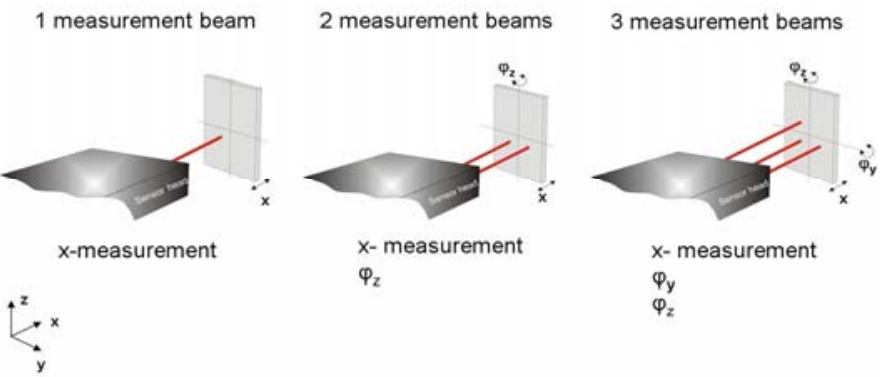

Fig. 4: Single, double and triple beam plane mirror interferometers

Figure 5 shows the photographs and the technical data of single, double and triple beam plan mirror interferometers. These interferometers have been developed by the Institute of Process Measurement and Sensor Technology in cooperation with the SIOS Meßtechnik GmbH. 


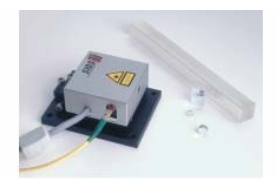

\section{Single Beam Interferometer}

Measurement range: $2 \mathrm{~m}$ mirror translation

rate: Resolution: $\quad 1 \mathrm{~nm} ; 0,1 \mathrm{~nm}$



Double Beam Interferometer

Measurement range : $2 \mathrm{~m}$

Resolution: $\quad 1 \mathrm{~nm} ; 0,1 \mathrm{~nm}$ Angular measure $\quad 2 \ldots 4, \geq 10 \mathrm{~mm}$ range: $\quad \pm 2$ arcmin Angular resolution: $\quad 0,1 \ldots, 0,01 \operatorname{arcsec}$ Angular measurement range with beam $\quad \pm 30$ arcmin

Fig. 5: Technical data of the plan mirror interferometers manufactured by SIOS Meßtechnik $\mathrm{GmbH}$

\section{SET-UP AND OPERATION OF THE NANOMEASURING MACHINE}

The nanomeasuring machine is shown in Figure 6.



Fig. 6: Nanomeasuring machine

Figure 7 illustrates the set-up of a nanomeasuring machine. The nanomeasuring machine consist of following main components:

- traceable linear and angular measurement instruments

- 3D-nanopositioning stage

- nanoprobes suitable for integration into nanomeasuring machine

- control equipment.

The nanomeasuring machine has been developed by the Institute of Process Measurement and Sensor Technology and manufactured by the SIOS Meßtechnik GmbH, Germany. The device implements sample scanning mode over a range of $25 \mathrm{~mm} \times 25 \mathrm{~mm} \times 5 \mathrm{~mm}$ with a resolution of $0,1 \mathrm{~nm}$. The measurement uncertainty is about $3 \mathrm{~nm}$. In sample scanning mode the sample being measured is moved and measured dimensionally and remains in contact with the fixed probe system, which acts as a zero indicator.

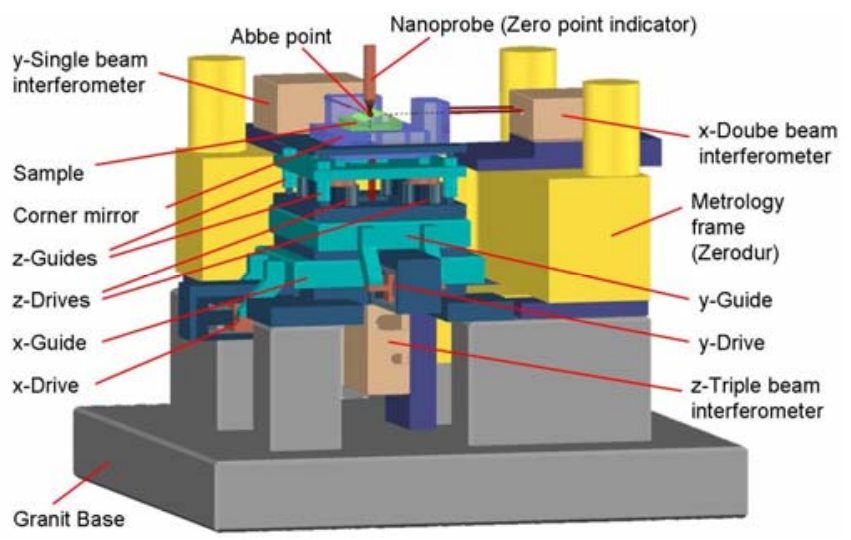

Fig. 7: Set-up of a nanomeasuring machine (sample scanning mode

Both the metrology frame, which carries the measuring systems (interferometers), and the 3-D stage are arranged on a granite base. The upper Zerodur ${ }^{\circledR}$ plate (not shown in Fig. 7) of the metrological frame is constructed such that different nanoprobes can be easily installed and removed. A corner mirror is moved by the 3-D stage, which is built in a stacked arrangement. The separate stages consist of ballbearing guides and voice coil drives [7]. The corner mirror is measured and controlled by single, double and triple beam plane mirror interferometers.

The excellent performance of the nanomeasuring machine is based on three important characteristics: (1) the Abbe error-free arrangement of the laser interferometers, (2) the application of an effective concept for compensating the stage errors and (3) the nanoprobes' acting as zero-point indicators only.

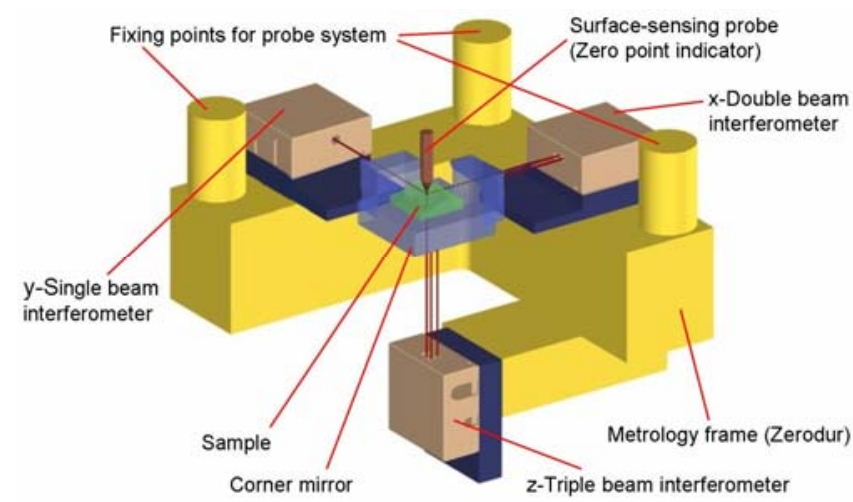

Fig. 8: Abbe offset-free design of the nanomeasuring machine

The three laser interferometer measuring beams are reflected off the outer surfaces of the corner mirror, whereby the virtual extension of the reflected beams intersect at the point of contact between the specimen and the sensor (see Fig. 8). Because sample scanning mode is implemented in 
the nanomeasuring machine, the Abbe comparator principle is realised over the entire measuring range. In this mode the probes only have the function of a zero-point indicator, which can be realised with very high precision.

Angular deviations of the guide systems are detected at the corner mirror by means of a double and a triple beam plane mirror interferometer. The detected angular deviations are compensated by a close-loop control system.

\section{ACKNOWLEDGMENTS}

The author wishes to thank all those colleagues who have contributed to the developments presented here. My special thanks go to the Thuringian Ministry of Science, Research and Arts for the promotion of nanocoordinate metrology in the framework of joint projects and the German Research Foundation (DFG) for funding the Collaborative Research Centre 622 "Nanopositioning and Nanomeasuring Machines" at the Technische Universität Ilmenau.

\section{REFERENCES}

[1] G. Wernicke; W. Osten: Holografische Interferometrie; VEB Fachbuchverlag Leipzig, 1. Auflage, 1982

[2] H. Haferkorn: Optik; VEB Deutscher Verlag der Wissenschaften Berlin, 1980

[3] Documents concerning the new definition of the meter; Metrologia 19, S. 163 - 177, 1984

[4] T.J. Quin: Practical realization of the definition of the metre; Metrologia 36 (3), S. $211-244,1997$

[5] H.-J. Büchner; G. Jäger: Plane mirror interferometer for precision lenght measurements; Proceedings of the euspenConference, Montepellier, France, S. 45 - 48, 2005

[6] H.-J. Büchner; G. Jäger: A novel plane mirror interferometer without using corner cube reflectors; Measurement Science and Technology 17, S. $746-752,2006$

[7] G. Jäger et al: A nanopositioning and nanomeasuring machine, operation, measured results; Nanotechnology and Precision Engineering; Vol. 2, S. 81 - 84, 2004 